International Journal of Pure and Applied Mathematics

Volume 83 No. 2 2013, 375-386

ISSN: 1311-8080 (printed version); ISSN: 1314-3395 (on-line version)

url: http://www.ijpam.eu

doi: http://dx.doi.org/10.12732/ijpam.v83i2.15

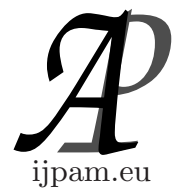

\title{
EXISTENCE OF AN OSCILLATING BEHAVIOR IN \\ THE DYNAMICS OF POPULATIONS MODELED BY \\ DELAY DIFFERENTIAL EQUATIONS WITH IMPULSES
}

\author{
V.I. Donev \\ Department of Mathematics \\ Technical University of Sliven \\ 8800, Sliven, BULGARIA
}

\begin{abstract}
This paper is dealing with the investigation of oscillating properties of delay differential equations with impulses, which model the dynamics of populations. A technique based on a special function called "impulsive exponent" is developed for construction of their characteristic equations. The resulting characteristic equations are examined for conditions under which the constructed solutions of the studied differential equations are found to be oscillating. The proposed method can be applied to solve practical problems in the field of the population dynamics, econometrics (dynamics of Goodwin's business cycles) etc.
\end{abstract}

AMS Subject Classification: 34A37, 93A30, 34K11, 34K45

Key Words: delay differential equation, impulse, oscillating solution, characteristic equation, impulsive exponent

\section{Introduction}

Many populations go through different stages of his life from the birth to the death. In order to find the general regularities in the lives of their different generations (the so-called oscillating behavior of the population), which are com-

Received: December 11, 2012

(c) 2013 Academic Publications, Ltd. url: www.acadpubl.eu 
monly observed in the nature, the population dynamic constructs and explores mathematical models. For this purpose, the researchers collect and analyze certain amounts of data at certain points of time. However, while physical processes such as acceleration does not take much time, the biological processes, such as pregnancy or the maturation of individuals, may take considerable time compared with the time, for which relevant laws are observed. Therefore, it is often necessary to bear in mind that some processes are started before the current time of the analysis of the data encoded in the mathematical model of the dynamics of the investigated population. Technologically, this is expressed as the argument is obliged to slow (to slow down in time) from the current point of time. The mathematical models that include such a formulation are called differential equations with deviating argument (DEDA). These equations provide an adequate mathematical tools for modeling processes and phenomena that depend on their prehistory (or even future). Indeed, many practical problems in various fields of science and technology are meaningless if they do not take into account the dependence of the described process on the previous status. Indicative of this is the work of N. Minorsky [5], which is devoted to the stabilization of the course of a ship and its automatic control, which clearly shows the need for a delay in the feedback mechanism.

In general, a differential equation with delayed argument has the form

$$
x^{\prime}(t)=f(x(t), x(t-h)), \quad h>0,
$$

where $x(t-h), h>0$ represents the solution in the recent past.

In biological terms, the dynamics of the different populations can be described by a model

$$
x^{\prime}(t)=B(x(t-h))-D(x(t)), \quad h>0
$$

where $x(t)$ is the size (the density) of some population, the function of birth $B($.$) is dependent on the gestation period, while the function of death D($. depends on the current status of the population. Of course, model (DM1) is seen under certain initial conditions, i.e.

$$
x(s)=\phi(s), \quad s \in[-h, 0] .
$$

One can identify the form of the differential model (DM1) in a variety of equations, modelling the dynamics of different populations and seen through the prism of different factors - the "restriction of food", "infection", "mate", "regeneration", "Ali's effect", etc. For example, the differential equation of Verhulst-Pearl

$$
x^{\prime}(t)=x(t) r\left[1-\frac{x(t)}{K}\right]
$$


models the dynamic of a population (e.g. bacteria, plankton, fish, mosquitoes, etc.) with a density $x(t)$ in the moment of time $t$. The species breeds in constant (nutritional) environment. Here, $r>0$ is called intrinsic growth rate of species $x(t), K>0$ is interpreted as the capacity of the environment for this type of species, while $r\left[1-\frac{x(t)}{K}\right]$ is the "per capita" growth rate of the species $x(t)$ in the moment of time $t$. Since there are fluctuations in the densities of the species around the "equilibrium point" K, then Hutchinson [4] suggested in the "per capita" growth rate to reflect the fact that the population growth of the previous generations (in terms of breeding) depends on the maturity of the species. This presumes an availability of time for the reproduction by a constant delay $h>0$, which characterizes the sexual maturity of the species. In such a way we get a logistic equation of Hutchinson, which is a generalization of the differential equation of Verhulst-Pearl:

$$
x^{\prime}(t)=x(t) r\left[1-\frac{x(t-h)}{K}\right], t \geq t_{0} .
$$

This is a first order differential equation with a delayed argument. In this equation, the growth rate becomes a natural context - namely it reveals the density of the functional dependence of the feedback mechanism, which needs certain units of time to respond to the changes in the density of the species $x(t)$. Historically, the equation (P1.1) is also used in 1935 in the economic theory of stability of business cycles (Goodwin). As a modification of (1.1) and at the same time as a realization of the differential model (DM1) in the sense of "mating" we can consider the differential equation of Nicholson's blowflies (1954)

$$
x^{\prime}(t)=P x(t-h) e^{\frac{-x(t-h)}{x_{0}}}-\delta\left(x(t), \quad t \geq t_{0},\right.
$$

where $\mathrm{P}$ is the maximum daily rate of egg production, $\delta$ is the daily rate of mortality and $x_{0}$ is the size of the population, in which it has a maximum reproduction.

Another example of the modification of (P1.1) and implementation of the differential model (DM1), but in the sense of "regeneration" is the differential equation of Haematopoiesis:

— modelled by Mackey and Glass (1977)

$$
x^{\prime}(t)=\frac{\lambda a x(t-h)}{a+x^{m}(t-h)}-g x(t), \quad t \geq t_{0},
$$

where $x(t)$ is the concentration of cells in the blood plasma unit currently at the time $t, g$ is the rate of blood loss, the positive coefficients $\lambda$ and $a$ are associated 
with a normal process of regeneration of blood cells from stem cells in the bone marrow and $h$ is the time needed to produce new blood cells;

— modelled by Wazewska-Czyzewska and Lasota (1976)

$$
x^{\prime}(t)=p e^{-\gamma x(t-h)}-\mu x(t) \quad, t \geq t_{0},
$$

where $x(t)$ is the concentration of cells in the blood plasma unit currently at the time $t, \mu$ is the possibility of blood loss, the positive coefficients $\gamma, p$ are associated with a normal process of regeneration of blood cells from stem cells in the bone marrow and $h$ is the time needed to produce new blood cells.

Meanwhile, the researchers have noted that a number of physical phenomena in their evolution have undergone short influences, which distort their natural course. For example, under the influence of catch or after the waste poisoning, the population size of the species $x(t)$ in the model (P1.1) (assume it is a fish) can be reduced dramatically for very short time intervals. An extension of the density of the population is possible for the migration of individuals from such another area. Similar phenomena occur in mechanics (movement of damper subjected to shock, effect of clock, speed variation of rocket separating its degree), in radio (generating units), in biology (work of the heart, growth of cells), etc. In the mathematical modelling of such phenomena and processes, for convenience it is naturally to ignore the short time duration of these effects and assume that the process changes its own state instantaneously and incrementally, i.e. with impulses. Moreover, the construction of mathematical models of these phenomena and processes generate so called Impulsive Differential Equations (IDE). Their solutions, generally speaking, are not only continuous functions, but also functions with discontinuities of first kind (see [6]).

So, if as a result of external impulsive influences at the moment $t=\tau_{k}$ the density of the species $x(t)$ from the model (P1.1) undergoes modification $\alpha_{k}$, then we can represent this by

$$
\Delta x\left(\tau_{k}\right)=x\left(\tau_{k}+0\right)-x\left(\tau_{k}-0\right)=\alpha_{k}, \quad k \in N
$$

where $x\left(\tau_{k}+0\right)$ and $x\left(\tau_{k}-0\right)$ are respectively the densities of the species before and after an impulse. Moreover, if the density has increased, then $\alpha_{k}>0$. If it has decreased, then $\alpha_{k}<0$.

Now, combining the theme of the feedback mechanisms (dependence on the prehistory) in (P1.1) with that of the impulsive effects in (P1.2), gives rise to Impulsive Differential Equations with Delay Argument (IDEDA). That 
is, (P1.1) and (P1.2) construct a first order delay differential equation with impulses of the form:

$$
\left\{\begin{array}{l}
x^{\prime}(t)=x(t) r\left[1-\frac{x(t-h)}{K}\right], \quad t \neq \tau_{k} \\
\Delta x\left(\tau_{k}\right)=x\left(\tau_{k}+0\right)-x\left(\tau_{k}-0\right)=\alpha_{k}, \quad k \in N
\end{array}\right.
$$

The model (P1) reveals the population dynamic of a species considered in the model of Hutchinson. Similar equations can be obtained on the model of Nicholson's blowflies, model of Lasota-Wazewska, etc. As mentioned above, (P1) can be used also in the econometrics in the study of business cycles (Goodwin).

Remark 1. After the classical "change of variable" $y(t)=\ln \left(\frac{x(t)}{K}\right)$ associated with the equilibrium point and after a convenient extra substitution, some differential equations modelled in sense of (DM1), such as (P1.1), can be transformed to the equation

$$
y^{\prime}(t)+p(t) y(t-h)=0, \quad t \neq \tau_{k}, t \geq t_{0},
$$

where the equilibrium point is now the trivial solution.

Remark 2. Considering the periodicity in the season, delivery of food, habits of the population, etc., it can be assumed that (P1) could model the population dynamics of the species $x(t)$ more precisely, if it is provided with constant (or periodic) coefficients, i.e. $p(t)=p=$ const., yielding the equation

$$
y^{\prime}(t)+p y(t-h)=0, \quad t \neq \tau_{k}, t \geq t_{0} .
$$

Assume further that the population experiences frequent "interference in its size" and these disturbances are modeled with impulsive conditions, which imitate the differential model, namely

$$
\Delta y\left(\tau_{k}\right)+u y\left(\tau_{k}-h\right)=0, \quad k \in N .
$$

Then (1.1) and (1.2) compose the first order delay differential equation with impulses

$$
\left\{\begin{array}{l}
y^{\prime}(t)+p y(t-h)=0, \quad t \neq \tau_{k}, t \geq t_{0} \\
\Delta y\left(\tau_{k}\right)+u y\left(\tau_{k}-h\right)=0, \quad k \in N
\end{array}\right.
$$

Remark 3. Various problems of the population dynamics, mathematical ecology, etc. in terms of oscillation, constraints and asymptotic stability of the solutions can be analyzed precisely with the help of the model (1.*). 


\section{Preliminary notes}

It is known on the qualitative theory of differential equations that the location of the roots of the characteristic equation $\quad \lambda+p e^{-\lambda h}=0 \quad$ completely defines the asymptotic stability of the trivial solution of (1.1). Moreover, the oscillation properties of (1.1) also depend on these roots. But unlike the equation without impulses (1.1), the problem of construction of the characteristic equation for $(1 . *)$ looks complicated, because of the loss of autonomy due to the presence of impulses. The monograph [1] is probably the only place where this is proposed for consideration. However, the characteristic system there for $(1 *)$ is built based on the assumption for positive coefficients and on a restriction to one of the system's parameters. In this work, the author using the ideas of [2] and [3] for "impulsive exponent", constructs a characteristic equations for the problem (1), which generalizes $(1 * *)$ and which has no restrictions about the coefficients or parameters. The resulting characteristic equations are examined for conditions under which the solutions of the studied differential equations are found to be oscillating. In this sense, the results obtained can be considered as complementary to and summarizing the results in [1].

Let the dependence of a process from its prehistory $h(t), h(t)<t$ is described by a first order delay differential equation of the form

$$
y^{\prime}(t)+r y(t)+p y(h(t))=0, t \neq \tau_{k}, k \in N, t \in J_{0}, J_{0}=\left[t_{0},+\infty\right) .
$$

Let's also the law of evolution of the process is connected with the division of points $\tau_{Y}=\left\{\tau_{i}\right\}_{i=1}^{+\infty}$, which are situated in $J_{0}$ and the following hypothesis is fulfilled:

$$
\begin{gathered}
\text { H1. } t_{0}=\tau_{0}<\tau_{1}<\tau_{2}<\ldots<\tau_{k}<\ldots, \lim _{k \rightarrow+\infty} \tau_{k}=+\infty \text {, and } \\
\max \left\{\tau_{k+1}-\tau_{k}\right\}<+\infty, k \in N ;
\end{gathered}
$$

The system of points $\tau_{Y}=\left\{\tau_{i}\right\}_{i=1}^{+\infty}$ represents the moments of time in which the observed process undergoes short disturbances, distorting its natural course. We call these points moments of impulse effects or "jump points" because in them the respective function-solution reveals its discontinuities of first kind as jumps. In order to manifest these jumps of the unknown function $y(t)$ we use the notation

$$
\Delta y\left(\tau_{k}\right)=y\left(\tau_{k}+\right)-y\left(\tau_{k}-\right)
$$

where

$$
y\left(\tau_{k}+\right)=y\left(\tau_{k}+0\right)=\lim _{t \rightarrow \tau_{k}+0} y(t), \quad y\left(\tau_{k}-\right)=y\left(\tau_{k}-0\right)=\lim _{t \rightarrow \tau_{k}-0} y(t)=y\left(\tau_{k}\right) .
$$


We assume the changes of the process are modelled by the following impulsive expression:

$$
\Delta y\left(\tau_{k}\right)+s y\left(\tau_{k}\right)+u y\left(h\left(\tau_{k}\right)\right)=0, \quad k \in N .
$$

Combining (*.1) and (*.2) we get the problem

$$
\left\{\begin{array}{l}
y^{\prime}(t)+r y(t)+p y(h(t))=0, t \neq \tau_{k}, \\
\Delta y\left(\tau_{k}\right)+s y\left(\tau_{k}\right)+u y\left(h\left(\tau_{k}\right)\right)=0, k \in N,
\end{array}\right.
$$

where the delay function $h(t)$ satisfies the hypothesis $\left(R^{+}=(0,+\infty)\right)$ :

H2. $h \in C^{1}\left(R^{+}, R^{+}\right), h^{\prime}(t)>0, h(t)<t, \lim _{t \rightarrow \infty} h(t)=+\infty$; while the coefficients satisfy the hypothesis:

H3. $r, p, s, u \in R$.

For our needs further assume that the location of the points of impulse effect for the solution $y(t)$ in terms of the delay function $h(t)$ are subject to the hypothesis: $\mathrm{xms}$

h1. $\exists n \geq 1, n \in N: h\left(\tau_{s}\right) \in\left\{\tau_{k}\right\}_{k=1}^{+\infty}, \tau_{s} \in\left\{\tau_{i}\right\}_{i=n+1}^{+\infty}$, i.e. $h\left(\tau_{s}\right)=$ $\tau_{s-j}, j \in N, s>j$.

Let we define the following key concepts:

Definition 1. Denote by $P A C_{\tau}^{1}(J)$ the set of all functions $u(t): R \rightarrow R$, such that:

(i) $u$ is a piece-wise absolutely continuously differentiable function on

$$
\left[\tau_{0}, \tau_{1}\right] \bigcup\left\{\left(\tau_{k}, \tau_{k+1}\right]\right\}_{1}^{\infty}
$$

(ii) $u$ is continuous from the left at $\tau_{k} \in \tau_{Y}$, i.e.

$$
u\left(\tau_{k-0}\right)=\lim _{t \rightarrow \tau_{k-0}} u(t)=u\left(\tau_{k}\right)
$$

iii) there exists a sequence of real numbers $\left\{u\left(\tau_{k+0}\right)\right\}_{1}^{\infty}$ :

$$
u\left(\tau_{k+0}\right)=\lim _{t \rightarrow \tau_{k+0}} u(t)
$$

iv) $u$ may have discontinuities of first kind at the points $\tau_{k} \in \tau_{Y}$, i.e.

$$
\Delta u\left(\tau_{k}\right)=u\left(\tau_{k}+\right)-u\left(\tau_{k}-\right), \quad k \in N
$$


that we characterize as down-jumps $\left(\Delta u\left(\tau_{k}\right)<0\right)$ or as up-jumps $\left(\Delta u\left(\tau_{k}\right)>0\right)$.

Definition 2. We say that the real valued function $x(t) \in P A C_{\tau}^{1}(J)$ is a solution of $(*)$, if it satisfies (*.1) for every $t \in\left(\tau_{k-1}, \tau_{k}\right), k \in N$ and satisfies the impulsive conditions (*.2) for $t \in \tau_{Y}=\left\{\tau_{i}\right\}_{i=1}^{\infty}$, as well.

Definition 3. The solution $x(t)$ of $(*)$ is said to be regular, if it is defined on $\left[T_{x},+\infty\right) \subseteq\left[\tau_{0},+\infty\right)$ and $\sup _{T \geq T_{x}}\{|x(t)|: t \geq T\}>0$.

Definition 4. We say that a function $u: J \rightarrow R$ has some property $Q$ finally (eventually) if there is a number $t_{(u, Q)} \in J$ such that $u(t)$ has this property $Q$ for every $t \geq t_{(u, Q)}$.

Definition 5. The regular solution $x(t)$ of $(*)$ is said to be non-oscillating if there exists a number $T_{1}>0$ such that $x(t)$ is of constant sign for every $t \geq T_{1}$. Otherwise, it is called oscillating.

Definition 6. We say that a function $u(t)$ has a form of an "impulsive exponent", if there exist numbers $A, \lambda \in R$, such that $u(t)$ can be written as

$$
u(t)=A^{i\left[\tau_{0}, t\right)} e^{-\lambda t}, \quad e=2,71828 \ldots,
$$

where $i\left[\tau_{0}, t\right)$ represents the number of moments of impulse effect $\tau_{k} \in \tau_{Y}$ for $u(t)$, situated in the interval $\left[\tau_{0}, t\right)$. Here, the constant $A \in R$ will be referred to as a "pulsatile coefficient" of the impulsive exponent. When $\lambda=0$, then $u(t)$ will be referred to as an impulsive line.

\section{Main Results}

In this study we examine the conditions for existence of oscillating solutions of a problem of type $(*)$, which has the form

$$
\left\{\begin{array}{l}
y^{\prime}(t)+r y(t)+p y(t-h)=0, t \neq \tau_{k} \\
\Delta y\left(\tau_{k}\right)+s y\left(\tau_{k}\right)+u y\left(\tau_{k}-h\right)=0, k \in N
\end{array}\right.
$$

The problem (1) represents a first order differential equation with impulses, with a (constant) delay argument $h(t)=t-h, h \in R^{+}$and constant coefficients, as well. We suppose that the hypotheses (h1), (H1)-(H3) are satisfied. Moreover, accordingly to the hypothesis (h1), when we have constant deviation argument, then $i[t-h, t)=L=$ const., $L \in N$. 
Analyzing the forms of the solutions of (1), when $s=u=0, h=0$, i.e. when we have an equation without delay and impulses, we presume that the relevant solutions of (1) have to be in an appropriate exponential and impulsive form. So, the result that follows will allow us to study the problem (1) for existence of oscillating solutions, based on the Definition 6 .

Lemma 1. The problem (1) admits a solution $y(t)$ in a form of an impulsive exponent if and only if the algebraic transcendental equation

$$
(r-\lambda)\left(1+\frac{(u r-s p)-u \lambda}{p}\right)^{L}+p e^{\lambda h}=0, L \in N
$$

possesses a root $\lambda \in R$.

Proof. $(=>)$ Let $y(t)$ be a solution of (1) in the form of an impulsive exponent, accordingly to Definition 6., i.e. $y(t)=A^{i\left[\tau_{0}, t\right)} e^{-\lambda t}$. Substituting $y(t)$ in (1) and taking into account that the considered functions are continuous from the left, we obtain:

- from the differential equation:

$$
\left(A^{i\left[\tau_{0}, t\right)} e^{-\lambda t}\right)^{\prime}+r A^{i\left[\tau_{0}, t\right)} e^{-\lambda t}+p A^{i\left[\tau_{0}, t-h\right)} e^{-\lambda(t-h)}=0 ;
$$

— from the impulsive conditions:

$$
\left(A^{i\left[\tau_{0}, \tau_{k}\right)} e^{-\lambda \tau_{k}}\right)(A-1)+s A^{i\left[\tau_{0}, \tau_{k}\right)} e^{-\lambda \tau_{k}}+u A^{i\left[\tau_{0}, \tau_{k}-h\right)} e^{-\lambda \tau_{k}} e^{\lambda h}=0 .
$$

After simple manuipulations, we obtain the system

$$
\left\{\begin{array}{l}
(r-\lambda) A^{i\left[\tau_{0}, t\right)}+p A^{i\left[\tau_{0}, t-h\right)} e^{\lambda h}=0, \\
A^{i\left[\tau_{0}, \tau_{k}\right)}(s+A-1)+u A^{i\left[\tau_{0}, \tau_{k}-h\right)} e^{\lambda h}=0 .
\end{array}\right.
$$

In accordance with the hypothesis (h1) we have $i[t-h, t)=L=$ const., $L \in N$. Then, let's replace $i\left[\tau_{0}, t\right)=n, i\left[\tau_{0}, t-h\right)=n-L$ when $t \in\left[\tau_{n}, \tau_{n+1}\right)$. In such a way, the last system takes the form

$$
\left\{\begin{array}{l}
(r-\lambda) A^{n}+p A^{n-L} e^{\lambda h}=0 \\
A^{n}(s+A-1)+u A^{n-L} e^{\lambda h}=0
\end{array}\right.
$$

or more precisely

$$
\left\{(r-\lambda) A^{L}+p e^{\lambda h}=0, A^{L}(s+A-1)+u e^{\lambda h}=0 .\right.
$$


The resulting system (2) has two variables: $A$ and $\lambda$. Here, we can rule out the variable $A$, with $A=1+\frac{(u r-s p)-u \lambda}{p}$ and the system (2) becomes equivalent to the equation

$$
(r-\lambda)\left(1+\frac{(u r-s p)-u \lambda}{p}\right)^{L}+p e^{\lambda h}=0,
$$

which in the sequel will be referred to as a characteristic equation of (1). Obviously if $y(t)$ is a solution of (1) in the form of an impulsive exponent, i.e. $y(t)=A^{i\left[\tau_{0}, t\right)} e^{-\lambda t}$, then $\lambda$ will satisfy the characteristic equation of (1) and $A=1+\frac{(u r-s p)-u \lambda}{p}$.

$(<=)$ Conversely, consider the characteristic equation (3) of (1) and assume that this equation possesses a root $\lambda \in R$. Then (1) admits a solution $y(t)$ designed by $\lambda$ and $A=1+\frac{(u r-s p)-u \lambda}{p}$ in the form of an impulsive exponent, accordingly to the Definition 6 .

The proof of the lemma is complete.

The next result provides conditions for existence of oscillating solutions of (1) in the mentioned above form of an impulsive exponent.

Theorem 1. The problem (1) admits an oscillating solution in a form of an impulsive exponent if and only if the algebraic transcendental equation (3):

(a) possesses a real root $\lambda>\frac{r u+p(1-s)}{u}$, when $u p>0$;

(b) possesses a real root $\lambda<\frac{r u+p(1-s)}{u}$, when $u p<0$.

Proof. (a) $(=>)$ From Lemma 1 we know that if $y(t)$ is a solution of (1) in the form of an impulsive exponent, i.e. $y(t)=A^{i\left[\tau_{0}, t\right)} e^{-\lambda t}$, then $\lambda \in R$ is a root of the characteristic equation (3) and $A=1+\frac{(u r-s p)-u \lambda}{p}$. But if this solution is oscillating, then $0>A=1+\frac{(u r-s p)-u \lambda}{p}$, i.e. $-1>\frac{(u r-s p)-u \lambda}{p}$, or $-1>\left(r-\frac{s p}{u}-\lambda\right) \frac{u}{p}$. From the last inequality we have $-\frac{p}{u}>r-\frac{s p}{u}-\lambda$, when $u p>0$. It does mean that $\lambda$ satisfies the relation $\lambda>\frac{r u+p(1-s)}{u}$.

$(<=)$ Conversely, let up $<0$ and suppose that the equation (3) possesses a root $\lambda$, where $\lambda>\frac{r u+p(1-s)}{u}$. Then $\lambda>r+\frac{p}{u}-\frac{s p}{u}$, from where it is seen that $-\frac{p}{u}>r-\frac{s p}{u}-\lambda$. It does mean that $-1>\left(r-\frac{s p}{u}-\lambda\right) \frac{u}{p}$, i.e. $0>$ $1+\left(r-\frac{s p}{u}-\lambda\right) \frac{u}{p}$. From the last inequality we can conclude that then (1) will admit a solution $y(t)$ in the form of an impulsive exponent, designed by $\lambda$ and $A=1+\frac{(u r-s p)-u \lambda}{p}$, accordingly to the Definition 6. Moreover, the "pulsatile coefficient" $A$ will be negative, because $0>1+\left(r-\frac{s p}{u}-\lambda\right) \frac{u}{p}$. Obviously, then 
the function $y(t)=A^{i\left[\tau_{0}, t\right)} e^{-\lambda t}$ will be an oscillating solution of (1) in the form of an impulsive exponent.

(b) The proof is analogous and respectively to (a). The proof of the theorem is complete.

The next theorem demonstrates results for existence of oscillating solutions of $\left(1^{*}\right)$, utilizing the achievements in Lemma 1 for the problem (1) when $r=$ $0, s=0$.

Theorem 2. The problem (1), when $r=0, s=0$, admits an oscillating solution $y(t)$ in a form of an impulsive exponent if and only if the algebraic transcendental equation

$$
-\lambda\left(1-\frac{u}{p} \lambda\right)^{L}+p e^{\lambda h}=0 .
$$

1) possesses a real root $\lambda>\frac{p}{u}$, when $p u>0$;

2) possesses a real root $\lambda<\frac{p}{u}$, when $p u<0$.

Proof. The proof is analogous to the proof of Theorem 1 and can be realized with simple substitution of $r=0, s=0$ in its statement.

The last theorem demonstrates results for existence of oscillating solutions of the differential equation with impulses

$$
\left\{\begin{array}{l}
y^{\prime}(t)+r y(t)=0, t \neq \tau_{k} \\
\Delta y\left(\tau_{k}\right)+s y\left(\tau_{k}\right)=0, k \in N
\end{array}\right.
$$

utilizing the achievements in Lemma 1 for the problem (1), when $p=0, u=0$.

Theorem 3. The problem (5) admits an oscillating solution in a form of an impulsive exponent if and only if $s<1$.

Proof. $(=>)$ Let $y(t)$ be a solution of (5) in the form of an impulsive exponent, accordingly to Definition 6., i.e. $y(t)=A^{i\left[\tau_{0}, t\right)} e^{-\lambda t}$. Substituting $y(t)$ in (1) and taking into account that the considered functions are continuous from the left, we obtain:

- from the differential equation:

$$
\left(A^{i\left[\tau_{0}, t\right)} e^{-\lambda t}\right)^{\prime}+r A^{i\left[\tau_{0}, t\right)} e^{-\lambda t}=0,
$$

- from the impulsive conditions:

$$
\left(A^{i\left[\tau_{0}, \tau_{k}\right)} e^{-\lambda \tau_{k}}\right)(A-1)+s A^{i\left[\tau_{0}, \tau_{k}\right)} e^{-\lambda \tau_{k}}=0 .
$$


After simple manuipulations, we obtain the system

$$
\left\{\begin{array}{l}
(r-\lambda) A^{i\left[\tau_{0}, t\right)}=0 \\
A^{i\left[\tau_{0}, \tau_{k}\right)}(s+A-1)=0 .
\end{array}\right.
$$

which will be referred to as a characteristic system of (5). One solution of the system obtained is $\lambda=r, A=1-s$. Obviously, if $y(t)$ is an oscillating solution of (5) in the form of an impulsive exponent and it is designed by $\lambda=r, A=1-s$, then we should have $A<0$. Therefore, $1-s<0$ is fulfilled, which means that then we have $s<1$.

$(<=)$ Conversely, let $s<1$. We can construct a solution $y(t)$ of $(5)$ in the form of a impulsive exponent, designed by $\lambda=r$ and $A=1-s$. Obviously, the "pulsatile coefficient" $A$ will be negative when $s<1$. Therefore, the function $y(t)=A^{i\left[\tau_{0}, t\right)} e^{-\lambda t}$ will be an oscillating solution of (5) in the form of an impulsive exponent.

The proof of the theorem is complete.

\section{References}

[1] D.D. Bainov, P.S. Simeonov, Oscillation Theory of Impulsive Differential Equations: Periodic solutions and Applications, International Publications, Orlando, Florida (1998).

[2] M.B. Dimitrova, V.I. Donev, Existence and asymptotic behavior of the positive solutions of first order neutral impulsive differential equations, Nonlinear Oscillations, 8, No. 3 (2005), 304-318.

[3] V.I. Donev, Existence and asymptotic behaviour of positive solutions of a class of neutral impulsive differential equations in special cases, In: Proc. 28 Int. Conf. on Appl. of Math. in Engin. and Econ., Sozopol (2002), Bulvest2000, Sofia (2003).

[4] G.E. Hutchinson, Circular Causal Systems in Ecology, Ann. N.Y. Acad. Sci (1948).

[5] N. Minorsky, Directional stability of automatically steered bodies, J. Amer. Soc. of Naval Engineers, 34 (1922), 280-309.

[6] S.G. Pandit, S.G. Deo, Differential Systems Involving Impulses, Springer Verlag, Berlin-New York (1982). 